\title{
Management of rectal chlamydia in an urban sexual health centre
}

\author{
L. Goodall
}

Cobridge Sexual Health Centre, Cobridge, Stoke on Trent, UK

\section{Introduction}

We reviewed management of rectal chlamydia in our clinic and adherence to 2015 BASHH audit standards'1.

\section{Methods}

Electronic patient records of 100 consecutive patients diagnosed with rectal chlamydia prior to 31 July 2016 were reviewed with respect to gender, sexuality, HIV status, symptoms, STI screening, treatment, test of cure and partner notification.

\section{Results}

Of the 100 patients whose notes were reviewed, $64 \%$ were female. All females were heterosexual. $94 \%$ of males (34/36) were MSM. 6 males (17\%) and no females were HIV positive. All males reported receptive anal intercourse. $89 \%$ of females $(57 / 64)$ reported anal intercourse.

14 patients attended as chlamydia contacts and a further 5 patients attended as contacts of gonorrhoea.

1 male patient presented with rectal symptoms (rectal pain). $23 \%$ of patients had other genital symptoms (figure 1).

Fig 1. Presenting symptoms of patients diagnosed with rectal chlamydia
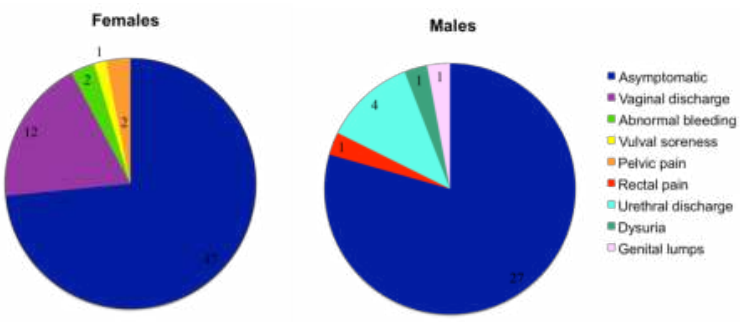

All patients were offered a full STI screen. All patients accepted full STI screening other than 2 females who declined blood tests and underwent testing for chlamydia and gonorrhoea only. $71 \%$ of patients had concomitant STIs (figure 2). 9 female patients $(14 \%)$ were diagnosed with rectal chlamydia in the absence of cervical chlamydia infection. 2 of these did not report anal intercourse.

Fig 2. Numbers of patients diagnosed with concomitant STIs
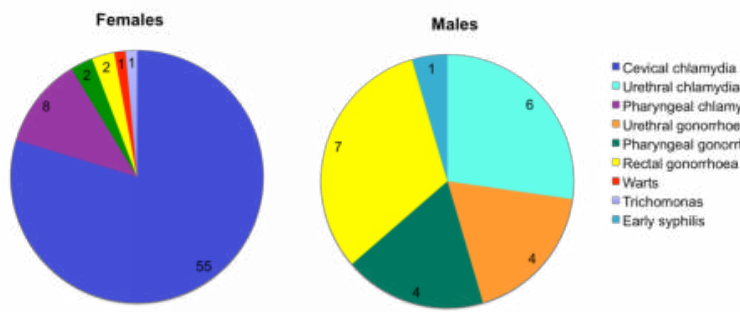
- Unetritral chlamydia - Pharyngeas chlamydily

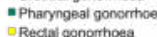
- Recalis gonomino - Warts ETichomonons
EEary syphilis
$24 \%$ (4 males and 20 females) were treated with azithromycin before being recalled for doxycycline. Reasons included; not initially tested for rectal infection, attendance as a chlamydia or gonorrhoea contact, initial treatment for a microscopy diagnosis of gonorrhoea. 20/24 (83\%) patients initially treated with azithromycin reported anal intercourse on sexual history taking.

Fig 3. Treatment regimes for patients with rectal chlamydia

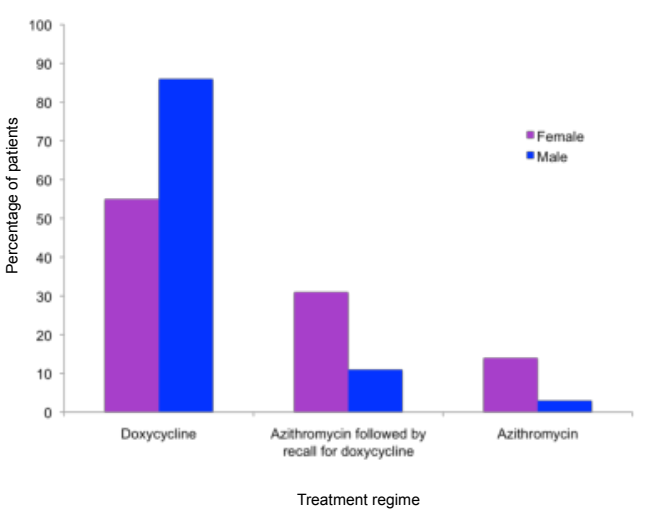

$35 / 36$ male $(97 \%)$ and $55 / 64$ female patients ( $86 \%$ ) were ultimately treated with doxycycline $100 \mathrm{mg}$ twice daily for at least 1 week. 1 male initially treated with azithromycin and ceftriaxone for presumed urethral gonorrhoea on microscopy did not return for follow-up. 9 females were treated with azithromycin $1 \mathrm{~g}$ stat; 2 were treated for introital chlamydia (not initially screened for rectal chlamydia) and did not return for doxycycline, 2 were treated as chlamydia contacts and did not return for doxycycline, 3 were pregnant, 1 was unable to tolerate doxycycline due to vomiting and 1 had a high risk of pregnancy and compliance concerns.

All patients were advised to attend for test of cure and $58 \%$ attended. Timing of tests of cure ranged from 3-11 weeks after treatment. All tests of cure were negative. 5 patients ( 4 females and 1 male) treated with azithromycin did not attend for test of cure despite several reminders.

All HIV positive patients were tested for LGV (1 LGV positive). 1 MSM who presented with rectal pain was not tested for LGV but a test of cure following treatment was negative.

Partner notification was performed in $99 \%$ of cases with $81 \%$ of traceable contacts reported as attended by the patient and $47 \%$ of traceable contacts verified as attended by the health advisor team.

\section{Discussion}

A large proportion of patients were issued with azithromycin as initial treatment for chlamydia despite reporting anal intercourse, necessitating recall for doxycycline. This is concerning, particularly in an era of increasing antibiotic resistance. Education sessions have been provided to the team, highlighting the importance of sexual history taking and use of doxycycline as first line chlamydia treatment when rectal infection is a possibility.

\section{Reference}

1. Nwokolo et al. 2015 UK national guideline for the management of infection with Chlamydia trachomatis. International Journal of STD \& AIDS 2016, vol. 27 251-267 